\title{
Diagnostics and analyses of decay process in laser produced tetrakis(dimethyl-amino)ethylene plasma
}

\author{
Guowen Ding, John E. Scharer, and Kurt L. Kelly \\ Department of Electrical and Computer Engineering, University of Wisconsin-Madison, \\ Wisconsin 53706-1687
}

(Received 3 January 2000; accepted 4 October 2000)

\begin{abstract}
A large volume (hundreds of $\mathrm{cm}^{3}$ ) plasma is created by a $193 \mathrm{~nm}$ laser ionizing an organic vapor, tetrakis(dimethyl-amino)ethylene (TMAE). The plasma is characterized as high electron density $\left(10^{13}-10^{12} \mathrm{~cm}^{-3}\right)$ and low electron temperature $(\sim 0.1 \mathrm{eV})$. To investigate the plasma decay processes, a fast Langmuir probe technique is developed, including detailed considerations of probe structure, probe surface cleaning, shielding, frequency response of the detection system, physical processes in probe measurement, dummy probe corrections as well as noise analysis. The mechanisms for the plasma decay are studied and a delayed ionization process following the laser pulse is found to be important. This mechanism is also supported by optical emission measurements which show that nitrogen enhances the delayed emission from TMAE plasma. A model combining electron-ion recombination and delayed ionization is utilized together with experimental results to order the terms and calculate the relaxation times for delayed ionization. The relaxation times are longer for lower TMAE pressures and lower electron densities. (C) 2001 American Institute of Physics. [DOI: 10.1063/1.1329154]
\end{abstract}

\section{INTRODUCTION}

Ionization of organic molecules with ultraviolet lasers is a new technique for generating large volume, homogeneous, well-defined plasmas. ${ }^{1}$ TMAE is a readily ionized organic gas, tetrakis(dimethyl-amino)ethylene, which can be single-photon ionized by a $193 \mathrm{~nm}$ laser, so that a large volume (hundreds of $\mathrm{cm}^{3}$ ), high initial plasma density $\left(>10^{13} \mathrm{~cm}^{-3}\right.$ ) plasma can be created. ${ }^{2-5}$ This technique has many potential applications, including a microwave reflector or absorber. 4,5

In this manuscript, the plasma parameters are primarily detected by a single Langmuir probe. However, difficulties lie in the special characteristics of TMAE plasma, including (1) high electron density $\left(10^{13}-10^{12} \mathrm{~cm}^{-3}\right)$ and low electron temperature $(\sim 0.1 \mathrm{eV}),(2)$ a rapid decay rate which requires a fast time response detection system and (3) probe contamination by TMAE vapor. The techniques of the fast Langmuir probe measurements including probe structure, probe surface cleaning, electromagnetic shielding, frequency response of the detection system, physical processes present in probe measurement interpretations, dummy probe corrections as well as noise analysis will be illustrated in detail in the experimental section.

The decay process of the laser-produced TMAE plasma is an important consideration for this technique. However, it is not as simple as atomic or small molecular ion plasma decay. In our experiment, we find that the apparent electronion recombination coefficient increases with time, and it increases more rapidly at higher TMAE pressures. However, the change in the recombination coefficient is only weakly dependent on electron temperature. This phenomenon as well as other experimental results cannot be interpreted by plasma diffusion, electron attachment, three-body recombi- nation with either electrons or neutrals as the third body, electron temperature effects, or multi-ion species processes. On the other hand, a new decay mechanism, the delayed ionization process, is indicated for the following reasons: (1) a delayed ionization mechanism provides the only good interpretation for all results; (2) the delayed optical emission experiments support this interpretation; (3) there are many reports on delayed ionization phenomena in laser photoionization experiments, and (4) an auto-ionization phenomenon in TMAE is also reported ${ }^{6}$ although no measurement of the relaxation time of the delayed ionization is given. Reasons (1) and (2) are presented in detail in this manuscript, and reasons (3) and (4) are supported by other works, which are discussed in later sections. Incorporating the delayed ionization process, a model for the decay processes is developed, and utilizing the experimental data the relaxation time of the delayed ionization is predicted.

\section{EXPERIMENT}

\section{A. Laser-produced TMAE plasma}

The TMAE plasma is created by a $193 \mathrm{~nm}$ laser photoionization of TMAE vapor in 2-16 $\mathrm{m}$ Torr pressure range. A schematic experimental arrangement is illustrated in Fig. 1. A laboratory plasma is created in a $50 \mathrm{~cm}$ long by $15 \mathrm{~cm}$ diameter cylindrical glass chamber, which is pumped to a base pressure of $5 \times 10^{-7}$ Torr by a diffusion pump. A Suprasil window which is transparent down to $180 \mathrm{~nm}$ is mounted on the laser entrance end of the vacuum chamber. On the opposite side of the chamber, a Langmuir probe and two flow feeds to introduce the TMAE vapor and nitrogen are inserted through feedthroughs to the vacuum chamber.

A Lumonics PM842 excimer laser runs in an ArF mode and produces $193 \mathrm{~nm}$ wavelength radiation. The half-width 


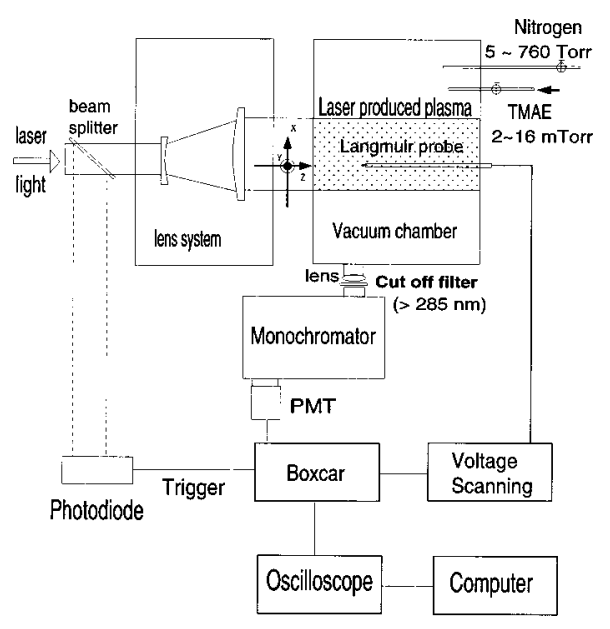

FIG. 1. Schematic of the experimental arrangement.

of the laser pulse is $20 \mathrm{nsec}$. The laser output cross section is $2.4 \times 3.2 \mathrm{~cm}^{2}$. The laser fluence is in the range of 4-8 $\mathrm{mJ} / \mathrm{cm}^{2}$. The laser attenuation length for $10 \mathrm{mTorr}$ TMAE is $1.7 \mathrm{~m}$. The laser beam is quite uniform, $\Delta I / I \leqslant 10 \%$ as measured by an apertured photo-diode. A calorimeter is used to accurately measure the laser beam intensity.

\section{B. Fast Langmuir probe diagnostics}

Probe structure: The plasma parameters are primarily detected by a single planar Langmuir probe, which consists of a double-shielded $50 \Omega$ coaxial cable connected to a tungsten wire. The radius of the probe for ion saturation current measurements is $0.5 \mathrm{~mm}$ and that for electron saturation current measurements is $0.25 \mathrm{~mm}$. The detailed probe structure is shown in Fig. 2. The tungsten wire probe is inserted through a hole of a four-bore alumina tube. In order to keep the probe surface clean, an $8 \mathrm{~cm}$ long half-loop of $0.15 \mathrm{~mm}$ diameter tungsten wire provides an indirect ohmic heating. The last hole is for a dummy probe wire, which is identical to the measurement probe wire, except that it is not exposed to the plasma. The exposed parts of the heating wire and the dummy probe are covered with a ceramic adhesive and a glass insulation layer to insulate them from the plasma.

Shielding and noise: Double-shielding and doubleisolating layers for the probe are shown in Fig. 2. For a rapidly pulsed plasma, the insulation and shielding for the Langmuir probe might not be perfect. We check the noise from the plasma wave penetration to the probe circuit by utilizing a dummy probe method. The dummy probe is an identical probe to the measurement probe, except that it is not exposed to the plasma. In this experiment, the signal from the dummy probe is always smaller than $0.1 \%$ of the electron saturation current and smaller than $10 \%$ of the ion saturation current obtained from the measurement probe. For the electron saturation current measurements, the noise from the plasma is neglected. For the ion saturation current measurements, the difference between measurement and dummy probe signals is regarded as the correct signal.

Fast response: A signal generator drives the probe tip to measure the frequency response of the detection system. The wave signals are varied from $200 \mathrm{MHz}$ to $20 \mathrm{kHz}$. When comparing the input from the generator and the output from data acquisition system, the relative errors for $20 \mathrm{kHz}$ to 2 $\mathrm{MHz}$ are $<0.5 \%$, those for $2 \mathrm{MHz}$ to $20 \mathrm{MHz}$ are $<1 \%$, and those for $20 \mathrm{MHz}$ to $200 \mathrm{MHz}$ slowly increase from $1 \%$ to $8 \%$. This is sufficient for our experimental delay time range of 0.1 to $2 \mu \mathrm{sec}$.

Probe measurement at early stage: There are many physical processes in a laser-produced plasma during the early stages. Langmuir probes can be used for accurate measurements after $t>100 \mathrm{nsec}$ but it is difficult to predict plasma parameters for earlier times $(t<100 \mathrm{nsec})$. This has been discussed in detail in our previous work. ${ }^{2}$ The laser pulse is typically extinguished in $35 \mathrm{nsec}$. When the laser is on, a probe sheath is forming, so the transient ion current, displacement current, as well as laser effects on the probe are expected to influence the probe current. After the laser is turned off, the laser effects are eliminated. The sheath response time is the order of $\omega_{p i}^{-1}$, where $\omega_{p i}$ is the plasma ion frequency, and $\omega_{p i}^{-1}=10 \mathrm{nsec}$ for $10^{12} \mathrm{~cm}^{-3}$ TMAE ion density. Then after several $\omega_{p i}^{-1}$, the transient ion current becomes very small. In the meantime, the probe sheath is formed, and the displacement current is small. Thus, all the data in this manuscript are measured after $t>100 \mathrm{nsec}$.

Probe sheath: The planar Langmuir probe in this experiment is a cross section of a Tungsten wire, of which the $\operatorname{radius}(r)$ is $0.5 \mathrm{~mm}$ for ion saturation current measurements. The probe area is $r^{2} \pi$, but the sheath around the probe can increase the probe current collection area to $2 \pi r \cdot \frac{1}{4} \cdot 2 \pi s$, where the sheath width $s$ can be calculated from the ChildLangmuir law. When the bias voltage is chosen as $1 \mathrm{~V}$, the correction of the sheath edge effect is not significant $(<20 \%)$. The sheath motion current due to the change of plasma parameters with time is very small $(<10 \%)$ when a

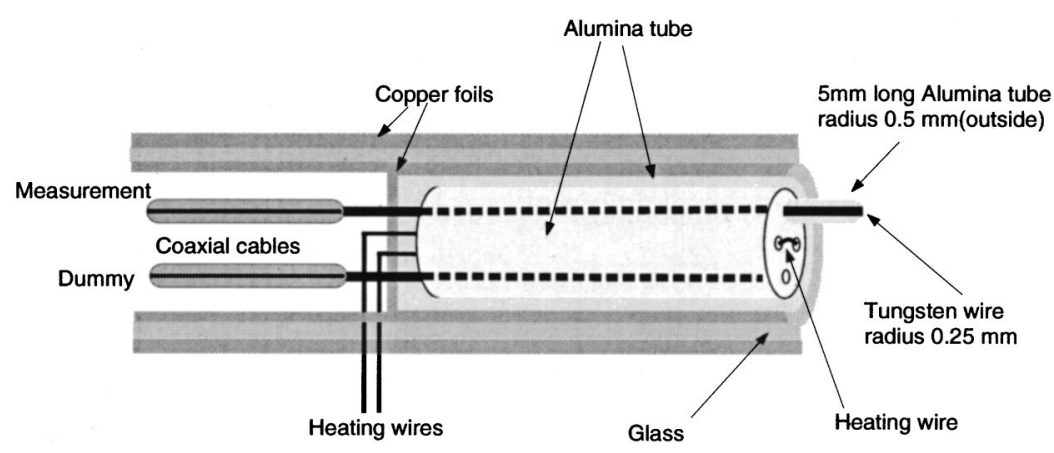

FIG. 2. Langmuir probe structure. 


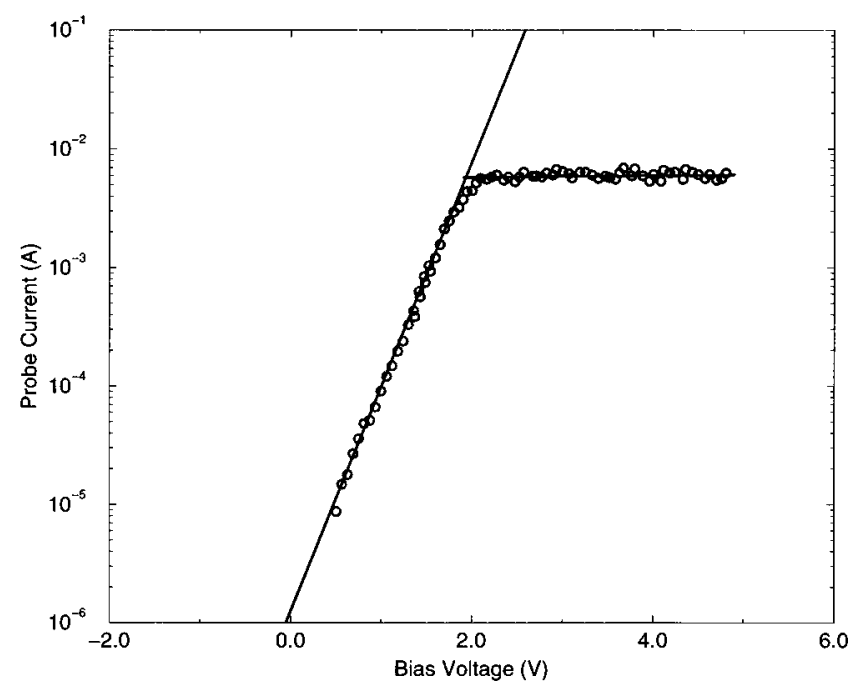

FIG. 3. Typical $I-V$ characteristic from Langmuir probe measurement, taken under $2 \mathrm{mTorr}$ TMAE pressure and $4 \mathrm{~mJ} / \mathrm{cm}^{2}$ laser fluence $200 \mathrm{nsec}$ after the laser shot.

small bias voltage is chosen, such as $1 \mathrm{~V}$. Electron saturation currents do not need these corrections since the Debye length is hundreds of times smaller than the probe size in this experiment.

Signal sampling: The Langmuir probe current-voltage characteristic curve is measured by a sampling technique with a boxcar, and the schematic arrangement is illustrated in Fig. 1. A sampling technique, triggered by the laser with a fixed delay, can be applied to the probe current, provided that the bias potential applied to the probe is slowly and linearly scanned. By such a technique, an $I-V$ characteristic can be displayed, which is considered equivalent to an instantaneous characteristic. The boxcar sampling window width $(\Delta \tau)$ is $20 \mathrm{nsec}$.

\section{Optical emission}

The plasma emission is measured by a monochromator at a right angle to the laser beam. A cutoff filter $(>285 \mathrm{~nm})$ is placed in front of the monochromator to eliminate the 193 $\mathrm{nm}$ laser light. A photo-multiplier tube (PMT) is connected to the monochromator, and its signal drives the boxcar, which provides the time-resolved emission spectra measurements. When a wavelength is fixed, and the PMT is connected to a fast Tektronix digital storage oscilloscope (Model TDS 350,1 GHz), the emission temporal profile at this wavelength is recorded. The data are typically averaged over 32 pulses. In this experiment, the temporal response time is $10 \mathrm{nsec}$, and the resolution of the monochromator is set to 4 $\mathrm{nm}$ in order to obtain good signal-to-noise ratios.

\section{RESULTS AND DISCUSSIONS}

\section{A. Electron density and temperature}

The decay process of the laser-produced TMAE plasma is primarily measured by a planar Langmuir probe. The typical Langmuir probe $I-V$ characteristic curve is shown in Fig. 3. The electron temperature is determined from the slope

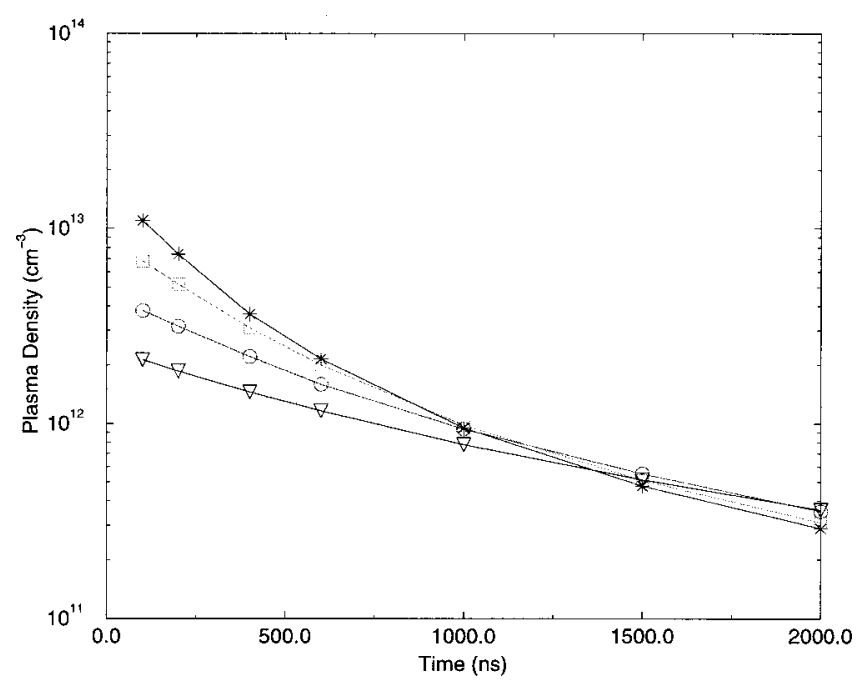

FIG. 4. Electron density versus time under conditions of $4 \mathrm{~mJ} / \mathrm{cm}^{2}$ laser fluence with TMAE pressures of (*) 16 mTorr, ( $\square) 8$ mTorr, (○) 4 mTorr, and $(\triangleleft) 2$ mTorr.

of the logarithmic $I-V$ characteristic, and the linear fit typically covers more than two decades of the probe signal values. The electron density is calculated from the electron saturation current $I_{e \text {,sat }}$, which is very flat with variations within $10 \%$. The electron saturation current is independent on the ion species which is very important for a molecular plasma. In theory, the ratio of ion to electron saturation current density, $J_{i, \text { sat }} / J_{e \text {, sat }}$, is not dependent on plasma density, electron temperature, or probe area, but only dependent on ion mass and negative ion proportion. In this experiment, the ratios $J_{i, \text { sat }} / J_{e \text {, sat }}$ are almost constant with time within $10 \%$ over $2 \mu \mathrm{sec}$. This implies that during the initial $2 \mu \mathrm{sec}$, only one dominant ion is present and the negative ion proportion is small. The electron density and temperature versus different delay times relative to the laser pulse are shown in Figs. $4-7$.

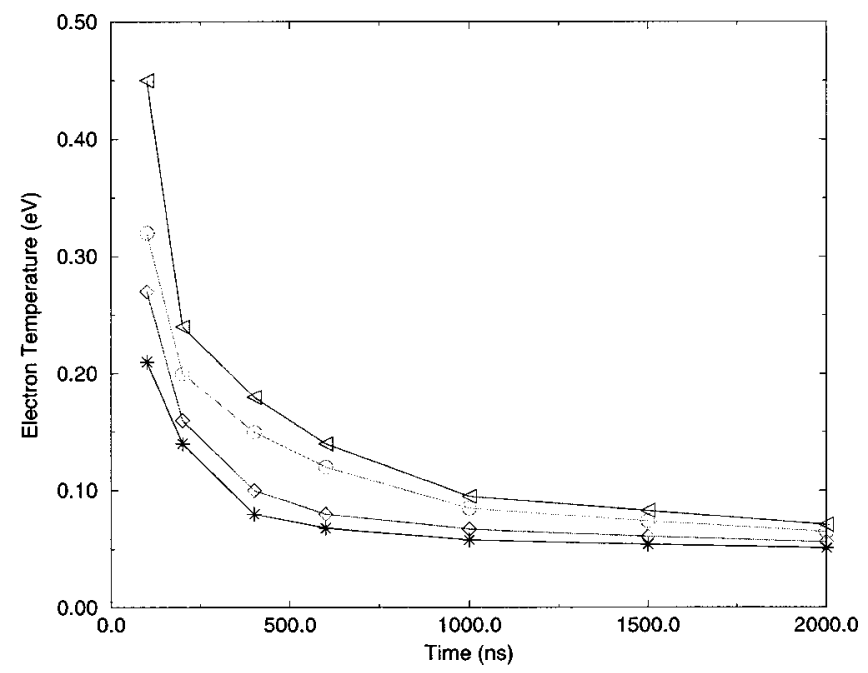

FIG. 5. Electron temperature versus time under conditions of $4 \mathrm{~mJ} / \mathrm{cm}^{2}$ laser fluence with TMAE pressures of $(*) 16 \mathrm{mTorr},(\diamond) 8 \mathrm{mTorr},(\bigcirc) 4 \mathrm{mTorr}$, and $(\triangleleft) 2$ mTorr. 


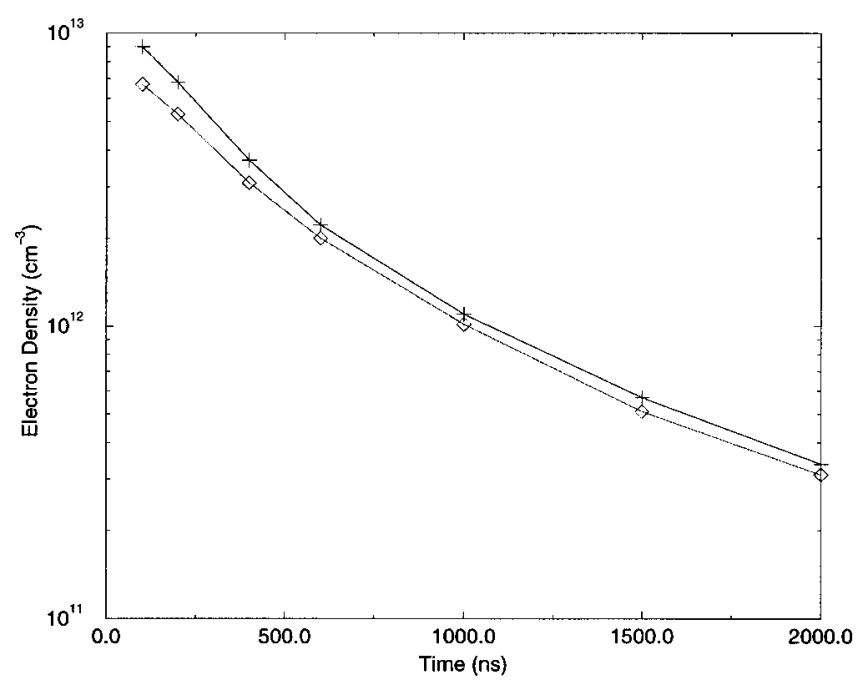

FIG. 6. Electron density versus time under conditions of 8 mTorr TMAE pressure with laser fluences of $(+) 8 \mathrm{~mJ} / \mathrm{cm}^{2}$ and $(\diamond) 4 \mathrm{~mJ} / \mathrm{cm}^{2}$.

The electron temperature, $T_{e}$, decays more rapidly at earlier times than at later times. The electron densities at earlier times are higher for both higher laser power and higher TMAE pressures, but for higher TMAE pressures, the electron density decays more rapidly.

Many experimental results are readily interpreted. The reason for such a low initial electron temperature is due to photo-ionization mechanisms. The electron temperature decay is due to electron-molecular collisions. The higher electron densities at earlier times for both higher laser power and higher TMAE pressure correspond to the initial higher ionized number density from the laser photo-ionization of TMAE.

\section{B. Analysis of plasma decay processes}

For a source-free plasma, the density decay process is usually described as

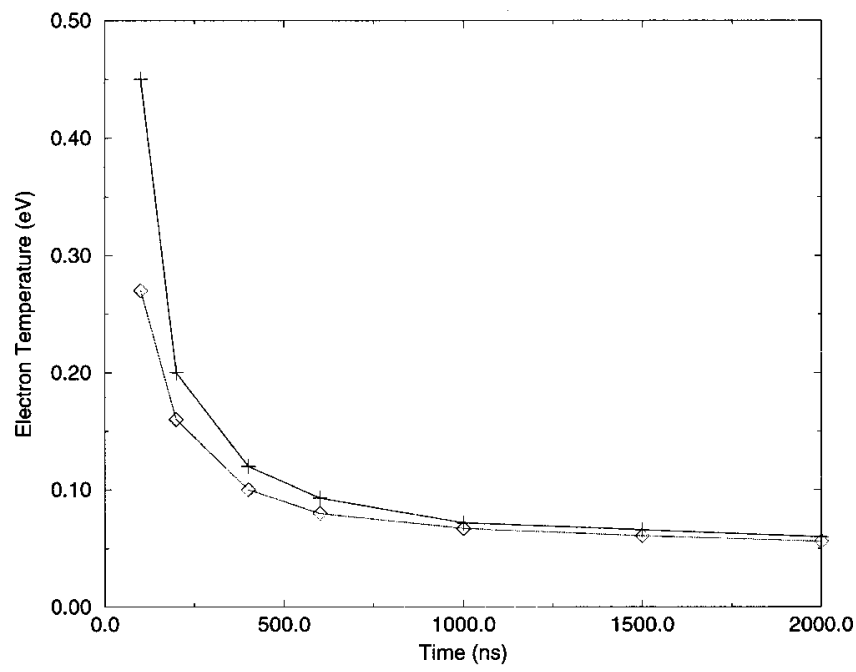

FIG. 7. Electron temperature versus time under conditions of $8 \mathrm{mTorr}$ TMAE pressure with laser fluences of $(+) 8 \mathrm{~mJ} / \mathrm{cm}^{2}$ and $(\diamond) 4 \mathrm{~mJ} / \mathrm{cm}^{2}$.

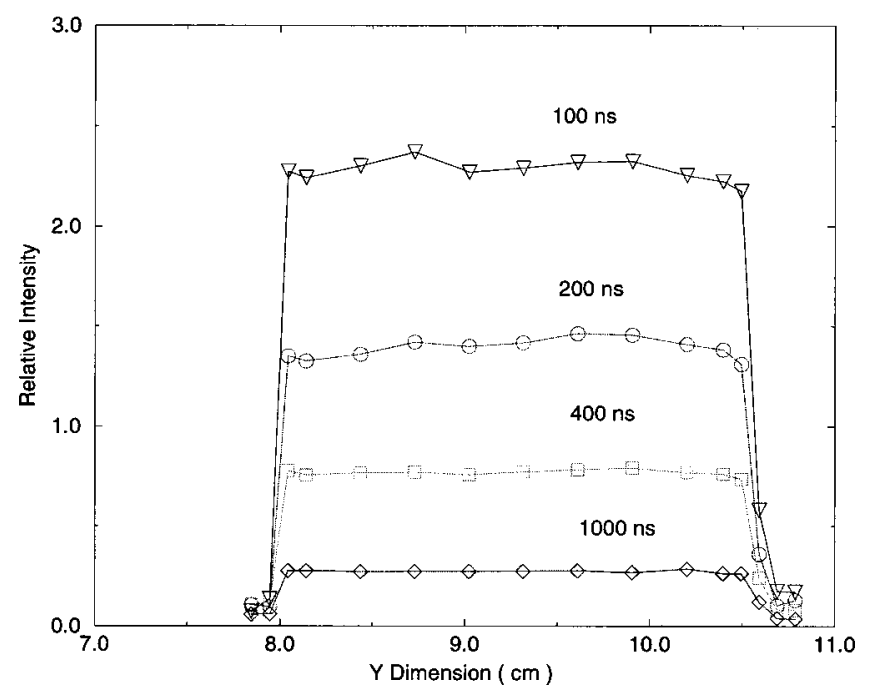

FIG. 8. Time-resolved plasma density profile in $y$ dimension.

$$
\frac{d n_{e}}{d t}=-D \nabla^{2} n_{e}-\alpha_{\mathrm{eff}} n_{e}^{2}-\nu_{a} n_{e},
$$

where $D$ is plasma diffusion coefficient, $\alpha_{\text {eff }}$ is the effective recombination coefficient, and $\nu_{a}$ is the electron attachment rate.

\section{Plasma diffusion}

For our large plasma volume, $\left(2.4 \times 3.2 \times 50 \mathrm{~cm}^{3}\right)$, the plasma diffusion process in the center is very small during the initial 2 sec period after the laser shot. The time-resolved density spatial profile is shown in Fig. 8, which shows that the diffusion is small in the center for $\mu$ sec time scales. The laser fluence over its output cross section is uniform, $\Delta I / I$ $\leqslant 10 \%$. It attenuates slightly along the propagation direction, and the fluence gradient is below $1 \% / \mathrm{cm}$ in this experiment, so that the electron density is correspondingly uniform initially. On the other hand, the plasma ambipolar diffusion is dependent on ion diffusion rather than on electron diffusion, and the maximum velocity of ion is the order of ion sound speed, ${ }^{7} \sqrt{k T_{e} / M_{i}}$, which means diffusion is important only on the order of $0.4 \mathrm{~mm}$ from the edge for $2 \mu \mathrm{sec}$ time scales. Thus, in the center of our large volume plasma $(2.4 \times 3.2 \times 50$ $\mathrm{cm}^{3}$ ), the diffusion process can be neglected for the initial microsecond time scale period.

\section{Electron attachment}

Since the TMAE molecule is a strong electron donor rather than an electron acceptor, the rate of electron attachment to TMAE should be small. Since the electron mobility in liquid TMAE is very large, ${ }^{8}$ this implies that the electron attachment rate to the TMAE molecule should be small. In this experiment, Langmuir probe measurements can determine if negative ions are present. Holmes et al. ${ }^{9}$ has given a formula to calculate the negative ion density,

$$
\frac{J_{e, \mathrm{sat}}}{J_{i, \mathrm{sat}}}=\frac{1}{0.6}\left(1-\frac{n_{-}}{n_{+}}\right) \sqrt{\frac{M_{i}}{2 \pi m_{e}}},
$$




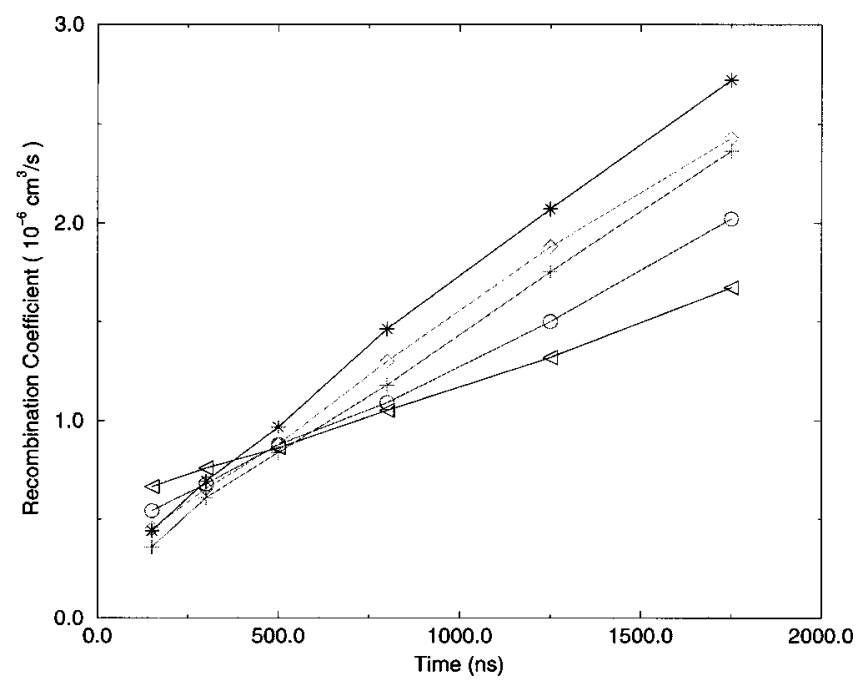

FIG. 9. Effective electron-ion recombination coefficient versus time under conditions of $4 \mathrm{~mJ} / \mathrm{cm}^{2}$ laser fluence with TMAE pressures of $(*) 16$ mTorr, $(\diamond) 8 \mathrm{mTorr},(\bigcirc) 4 \mathrm{mTorr}$, and $(\triangleleft) 2 \mathrm{mTorr}$, and under $8 \mathrm{~mJ} / \mathrm{cm}^{2}$ laser fluence with a TMAE pressure of $(+) 8$ mTorr.

where $M_{i}$ is the positive ion mass, $m_{e}$ is the electron mass, $n_{-}$and $n_{+}$are the negative and positive ion density, respectively, and $J_{e \text {,sat }}$ and $J_{i \text {,sat }}$ are electron and ion saturation current densities. If negative ion formation is important, $n_{-} / n_{+}$should increase with time due to rapidly decaying in $n_{+}$and increasing in $n_{-}$from zero, so that the electron and ion saturation current density ratio $J_{e \text {,sat }} / J_{i \text {, sat }}$ should decrease with time. However, no decreasing trend of the ratio $J_{e, \text { sat }} / J_{i \text {,sat }}$ with time can be found within $10 \%$ over $2 \mu \mathrm{sec}$. Thus, the electron attachment effect must be very small for our experimental conditions over the initial $2 \mu \mathrm{sec}$.

\section{Electron-ion recombination}

With the neglect of both diffusion and electron attachment processes, which are small in the experiment, Eq. (1) becomes

$$
\frac{d n_{e}}{d t}=-\alpha_{\mathrm{eff}} n_{e}^{2}
$$

The solution to this equation is

$$
\frac{1}{n_{1}}=\frac{1}{n_{0}}+\int_{t_{0}}^{t_{1}} \alpha_{\text {eff }} d t
$$

where $n_{0}$ and $n_{1}$ are the electron densities at times $t_{0}$ and $t_{1}$, respectively. The effective $\alpha_{\text {eff }}$ in this manuscript are approximately calculated from the corresponding electron densities at two successive measurement times:

$$
\alpha_{\text {eff }}=\left(\frac{1}{n_{1}}-\frac{1}{n_{0}}\right) /\left(t_{1}-t_{0}\right) \text {. }
$$

Using the data in Figs. 4 and 6, we plot $\alpha_{\text {eff }}$ versus time for different experimental conditions in Fig. 9. The results show that $\alpha_{\text {eff }}$ is not constant with time, but increasing. The higher the TMAE pressure, the more rapid the increase in $\alpha_{\text {eff }}$ with time.

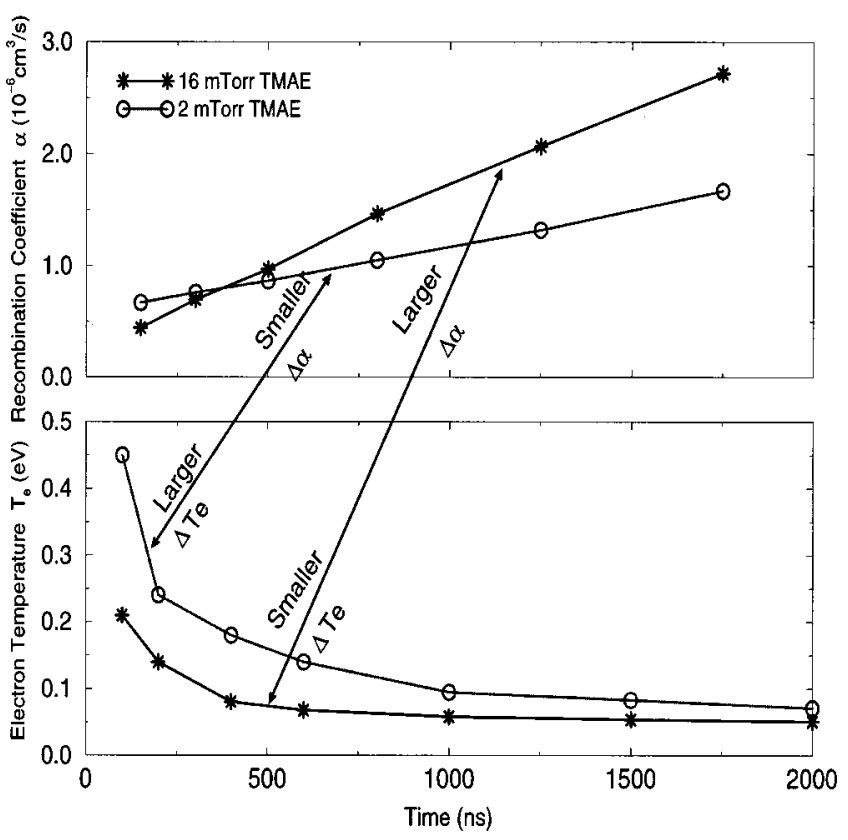

FIG. 10. Comparison of the variation of $\alpha_{\mathrm{eff}}$ and $T_{e}$ with time under conditions of $4 \mathrm{~mJ} / \mathrm{cm}^{2}$ laser fluence with TMAE pressures of 2 and $16 \mathrm{mTorr}$.

Another result is that the change in $\alpha_{\text {eff }}$ is only weakly dependent on the change in electron temperature. If the electron temperature $T_{e}$ effect was important in the change in $\alpha_{\text {eff }}$, a larger $\left|\Delta T_{e}\right|$ should cause a larger change in $\alpha_{\text {eff }}$, but the experimental results in Fig. 10 show the opposite relation. First, from $t=100 \mathrm{nsec}$ to $t=2000 \mathrm{nsec},\left|\Delta T_{e}\right|$ for the 2 mTorr TMAE case is larger than that in the 16 mTorr case, but $\left|\Delta \alpha_{\text {eff }}\right|$ in the 2 mTorr case is smaller than that of 16 mTorr case. Second, for the 16 mTorr TMAE pressure case, $T_{e}$ is almost constant in time $(0.6 \mu \mathrm{sec}<t<2 \mu \mathrm{sec})$, but the $\alpha_{\text {eff }}$ is approximately tripled. These results show that the change in $\alpha_{\text {eff }}$ is only weakly dependent on the change in electron temperature. This agrees with a general rule ${ }^{10}$ that for a larger molecular ion, $\alpha_{\text {eff }}$ is less electron temperature dependent, e.g., $\mathrm{H}_{3} \mathrm{O}^{+}\left(\mathrm{H}_{2} \mathrm{O}\right)_{2}$ and $\mathrm{NH}_{4}^{+}\left(\mathrm{NH}_{3}\right)_{2}$ plasmas ${ }^{11,12}$ which are almost independent of $T_{e}$.

The experimental results cannot be interpreted by the three-body recombination and muti-ion species effects:

(1) The process of three-body recombination with the electron as the third body (ion $+\mathbf{e}+\mathrm{e}$ ): This process causes $\alpha_{\text {eff }}$ to decrease as the electron density decays. This is contrary to the observation that the $\alpha_{\text {eff }}$ increases as the electron density decays.

(2) The process of three-body recombination with the TMAE molecule as the third body (ion+e+TMAE): The TMAE density is constant with time, so this process cannot interpret the increase of $\alpha_{\text {eff }}$ with time. In addition, to our knowledge, there are no reports of such a three-body process being important in the mTorr scale range.

(3) The multi-ion species effect: This effect must cause $\alpha_{\text {eff }}$ to decrease with time. If two components are assumed, the component with a larger $\alpha_{j}$ decays more rapidly, so the proportion of the component with the larger $\alpha_{j}$ must 
decrease with time. As a result, the global $\alpha_{\text {eff }}$ must decrease with time. This is in conflict with the experimental results of $\alpha_{\text {eff }}$ increasing with time. In addition, the ratios $J_{i \text {, sat }} / J_{e \text {, sat }}$ from the Langmuir probe measurement are constant within the experimental error $(10 \%)$, which implies that over the initial $2 \mu \mathrm{sec}$, the ion species mix does not change.

Thus, the experimental observations cannot be interpreted by the factors discussed here. This implies that another physical process is important in the decay process of the laser-produced TMAE plasma.

\section{Delayed ionization}

Photons absorbed by TMAE molecules may not contribute to direct ionization. When the TMAE molecule is excited by the laser photon $(6.4 \mathrm{eV})$, the total energy (electronic + vibrational+rotational) exceeds the first ionization potential (5.4 eV for TMAE), but some of those molecules are still neutral. Such an excited state is called a super-excited state. ${ }^{13}$ This phenomenon has been found in many molecules, including TMAE. ${ }^{6}$ For some of them, the lifetime can be hundreds of nsec, even tens of $\mu \mathrm{sec}$, and the most prominent example is $C_{60} \cdot{ }^{14,15}$ There are also many reports for other molecules such as benzene, ${ }^{16-19}$ benzene clusters, ${ }^{20,21}$ benzene-noble gas dimers, ${ }^{22}$ naphthalene, ${ }^{23}$ azulene, ${ }^{24}$ triethylamine, ${ }^{25}$ ketene, $^{26} \mathrm{Nb}$, Ta, and $\mathrm{W}$ clusters, ${ }^{27,28}$ $C_{70}{ }^{14,29}$ fullerenes $C_{n}(n<96),{ }^{30}$ etc. There are many theoretical articles, too. ${ }^{31,32}$ The experiments and theories are summarized in review articles. ${ }^{33-36}$

When a molecule is excited by a photon with energy higher than its ionization potentials (IP), it does not necessarily promptly ionize, particularly for a large molecule. ${ }^{37}$ The physical processes are illustrated as follows: ${ }^{35,13,38}$

$A B+$ photon $\rightarrow\left\{\begin{array}{l}A B^{+}+e(\text { direct ionization }) \\ A B^{* *} \rightarrow\left\{\begin{array}{l}A B^{+}+e(\text { delayed ionization }) \\ A+B(\text { dissociation })\end{array}\right.\end{array}\right.$

where $A B^{* *}$ represents molecule $A B$ in a super-excited state.

The analysis suggests that delayed ionization could occur in $193 \mathrm{~nm}$ laser photo-ionization of TMAE. There are several arguments to support this interpretation.

First, the delayed ionization mechanism can interpret the "unexplained" experimental observations. (1) The delayed ionization decays rapidly with time, so it becomes less important during later times. This causes $\alpha_{\text {eff }}$ to increase with time. (2) As the TMAE pressure increases, due to the collisional reaction $\mathrm{TMAE}+\mathrm{TMAE}^{* *}, \mathrm{TMAE}^{* *}$ decays more rapidly, so that the delayed ionization process is reduced. This is the reason that the plasma of 16 mTorr TMAE case decays more rapidly than that of 2 mTorr TMAE case.

Second, the delayed optical emission experiments strongly support the delayed ionization interpretation, which will be presented in the next section (Sec. III D).

Third, there are many reports on delayed ionization phenomenon in laser ionization of large molecules. ${ }^{14-36}$

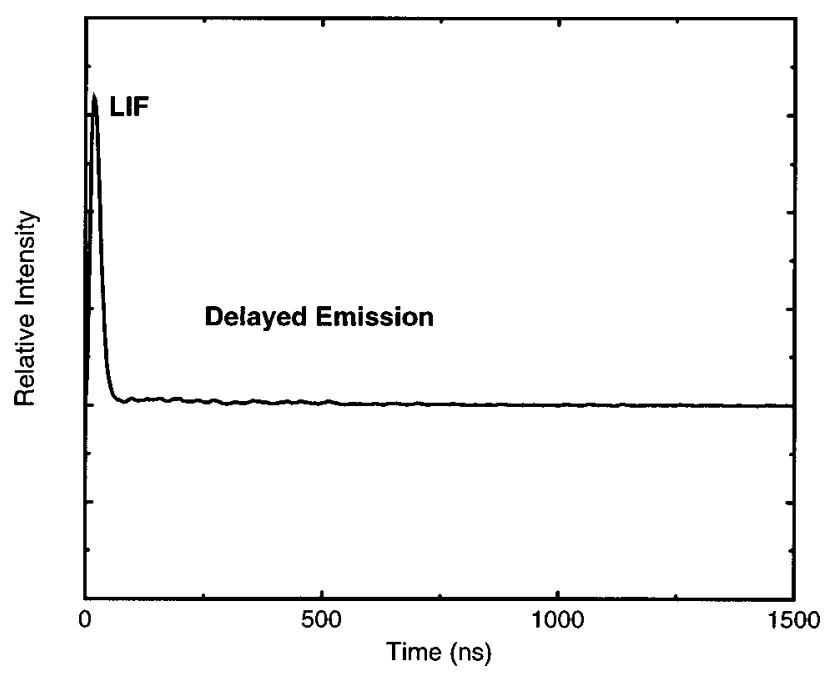

FIG. 11. The emission temporal profile from laser-produced TMAE plasma measured at $480 \mathrm{~nm}$.

Fourth, the TMAE super-excited phenomenon has been reported $^{6}$ when excited from 185 to $196 \mathrm{~nm}$, although the relaxation time of the delayed ionization is not measured. According to this report, when TMAE is excited by a 193 nm laser, superexcited TMAE** can be created.

In sum, the mechanisms of the laser-produced TMAE plasma decay processes are studied, compared with the experimental measurements, and a delayed ionization process is indicated.

\section{Optical emission measurement}

A spectrometer at a right angle to the laser beam is utilized to collect the plasma emission. The temporal emission intensity at $480 \mathrm{~nm}$ with a $4 \mathrm{~nm}$ resolution is shown in Fig. 11. The first peak is primarily a response to laser induced fluorescence (LIF). For later times, such as $t>100 \mathrm{nsec}$, the LIF emission is greatly reduced. Interesting results occur when 5, 50, and 760 Torr of nitrogen are added to 8 mTorr of TMAE. The emission spectra intensity for $300 \mathrm{nsec}<t$ $<1300 \mathrm{nsec}$ is increased with increasing nitrogen pressure, as shown in Fig. 12. The relative spectra are very similar for all cases, which implies that the majority species has not changed during this period. The peak of $480 \mathrm{~nm}$ has been reported as an emission for a TMAE Rydberg state (R1) which has $20 \mathrm{nsec}$ radiative lifetime. ${ }^{39,40}$ Since nitrogen does not react with TMAE in the ground state, ${ }^{41}$ and since nitrogen does not absorb the $193 \mathrm{~nm}$ photon, the enhancement of the delayed emission intensity suggests that there should be an energy-storage mechanism during the laser shot, and energy is released during the delay process. The process of the energy storage should involve a long-lifetime (hundred of nanosecond time scale) and highly excited species (above the first TMAE Rydberg state energy). This species is the source for the enhanced delayed emission.

In sum, the results of the enhancement of the delayed emission from a TMAE plasma by nitrogen suggests that a long-lifetime (hundred of nanosecond time scale) highly excited state(above the first TMAE Rydberg state energy) is 


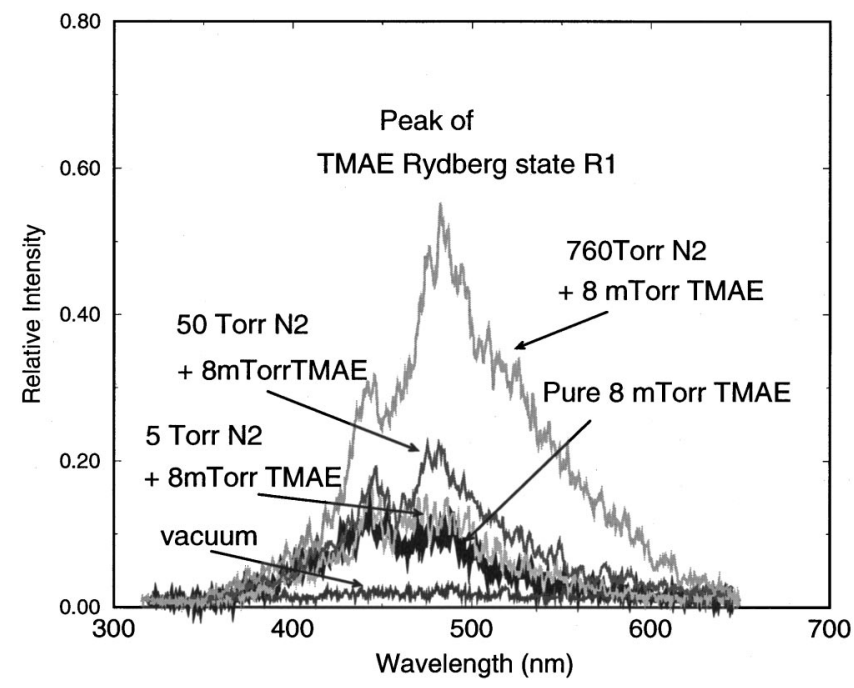

FIG. 12. Nitrogen effect on delayed emission from the laser produced TMAE plasma emission spectra, measured during the time window of $300 \mathrm{nsec}<t<1300 \mathrm{nsec}$ after the laser pulse.

important in the TMAE plasma, which could be a source for the delayed ionization. This result supports the delayed ionization mechanism.

\section{MODEL}

It is challenging to estimate the relaxation time of the delayed ionization process due to the many physical and chemical processes involved. However, the laser-produced TMAE plasma has some excellent properties which reduce the number of those processes. In this experiment, diffusion and electron attachment processes are negligible due to the arguments discussed in Sec. III. The other three process (three-body recombination with either an electron or neutral as the third body, electron temperature effects or multi-ion species processes) have been discussed and shown to be inadequate for interpreting the experimental results in Sec. III. Thus it is reasonable to assume that the change in the electron density is primarily due to the electron-ion recombination process, and the delayed ionization process. So the time derivative of electron density is governed by

$$
\frac{d n_{e}}{d t}=-\alpha n_{e}^{2}+D(t),
$$

where $n_{e}$ is the electron density, $\alpha$ is electron-ion recombination rate, and $D(t)$ is the delayed ionization rate (or electron density created by the delayed ionization in a unit time), which should decay with time.

Substituting $d n_{e} / d t=-\alpha_{\text {eff }} n_{e}^{2}$ from Eq. (3) into this equation, we obtain

$$
D(t)=\left(\alpha-\alpha_{\mathrm{eff}}\right) n_{e}^{2} .
$$

The physical meaning of $\alpha-\alpha_{\text {eff }}$ is the change in the effective recombination coefficient due to the delayed ionization process.

The relaxation time, $\tau$, of the delayed ionization can be defined as

$$
\tau=\frac{-1}{d \ln D(t) / d t} .
$$

We substitute Eq. (8) into Eq. (9), and neglect $d \ln (\alpha$ $\left.-\alpha_{\text {eff }}\right) / d t$. Thus the relaxation time $\tau$ can be calculated approximately as

$$
\begin{aligned}
\tau & =\frac{-1}{d \ln \left[\left(\alpha-\alpha_{\mathrm{eff}}\right) n_{e}^{2}\right] / d t} \\
& =\frac{-1}{d \ln \left(\alpha-\alpha_{\mathrm{eff}}\right) / d t+d \ln \left(n_{e}^{2}\right) / d t} \approx \frac{-1}{d \ln \left(n_{e}^{2}\right) / d t} .
\end{aligned}
$$

Here the approximation is valid if

$$
\left|\frac{d \ln \left(\alpha-\alpha_{\mathrm{eff}}\right)}{d t}\right| \ll\left|\frac{d \ln \left(n_{e}^{2}\right)}{d t}\right| .
$$

Since $\quad\left|d \ln \left(\alpha-\alpha_{\text {eff }}\right) / d t\right|=\left|\left(d \alpha / d t-d \alpha_{\text {eff }} / d t\right) /\left(\alpha-\alpha_{\text {eff }}\right)\right|, \quad$ the validation of Eq. (10) needs two conditions, namely,

$$
|d \alpha / d t| \ll\left|d \alpha_{\text {eff }} / d t\right|
$$

and

$$
\left|\frac{d \alpha_{\text {eff }} / d t}{\left(\alpha-\alpha_{\text {eff }}\right)}\right| \ll\left|\frac{d \ln \left(n_{e}^{2}\right)}{d t}\right| .
$$

The assumption $|d \alpha / d t| \ll\left|d \alpha_{\text {eff }} / d t\right|$ requires that the electron-ion recombination coefficient $\alpha$ change much less rapidly than the effect caused by the delayed ionization rates. In Sec. III B 2, the change in $\alpha$ due to the electron temperature and three-body effects has been shown to be inadequate for interpretation of the experimental results for the change in $\alpha_{\text {eff }}$, but only the delayed ionization mechanism provides a good interpretation. This means that the change of $\alpha$ with time $(d \alpha / d t)$ due to any reason other than the mechanism of delayed ionization is much smaller than $d \alpha_{\text {eff }} / d t$ due to the mechanism of delayed ionization, or $|d \alpha / d t| \ll\left|d \alpha_{\text {eff }} / d t\right|$.

Equation (12) can be verified by substituting the relevant parameters, which are all measured in the experiment except the $\alpha$ value. To prove the validity of Eq. (12), $\alpha$ is estimated by the effective $\alpha_{\text {eff }}$ measured in this work. The effective $\alpha_{\text {eff }}$ is a combination of electron-ion recombination ( $\alpha$ value) and delayed ionization contributions. Since $|d \alpha / d t|$ $\ll\left|d \alpha_{\text {eff }} / d t\right|$, with the delayed ionization time scale increasing, its contribution to the $\alpha_{\text {eff }}$ value decreases with time. Thus we conclude that the $\alpha$ value is larger than $\alpha_{\text {eff }}$, and they become closer for later times when the delayed ionization processes is less important. Based on this conclusion, we can estimate $\alpha$ from the effective $\alpha$ eff that we have measured.

For the 16 mTorr TMAE case, $\alpha_{\text {eff }}$ increases from 2.1 $\times 10^{-6} \mathrm{~cm}^{3} / \mathrm{sec}$ at $t=1250 \mathrm{nsec}$ to $2.8 \times 10^{-6} \mathrm{~cm}^{3} / \mathrm{sec}$ at $t$ $=1750 \mathrm{nsec}$. Since $\alpha_{\text {eff }}$ increases with time, $\alpha_{\text {eff }}>3$ $\times 10^{-6} \mathrm{~cm}^{3} / \mathrm{sec}$ is predicted for later times. Thus, $\alpha>\alpha_{\text {eff }}$ $>3 \times 10^{-6} \mathrm{~cm}^{3} / \mathrm{sec}$ is predicted, based on the conclusion that the $\alpha$ value is close to but larger than $\alpha$ eff for later times. The analysis here does not imply that the $\alpha$ value is around 3 $\times 10^{-6} \mathrm{~cm}^{3} / \mathrm{sec}$, but the $\alpha$ value could be larger, such as 4 $\times 10^{-6} \mathrm{~cm}^{3} / \mathrm{sec}$ or more. A more accurate $\alpha$ value is difficult 

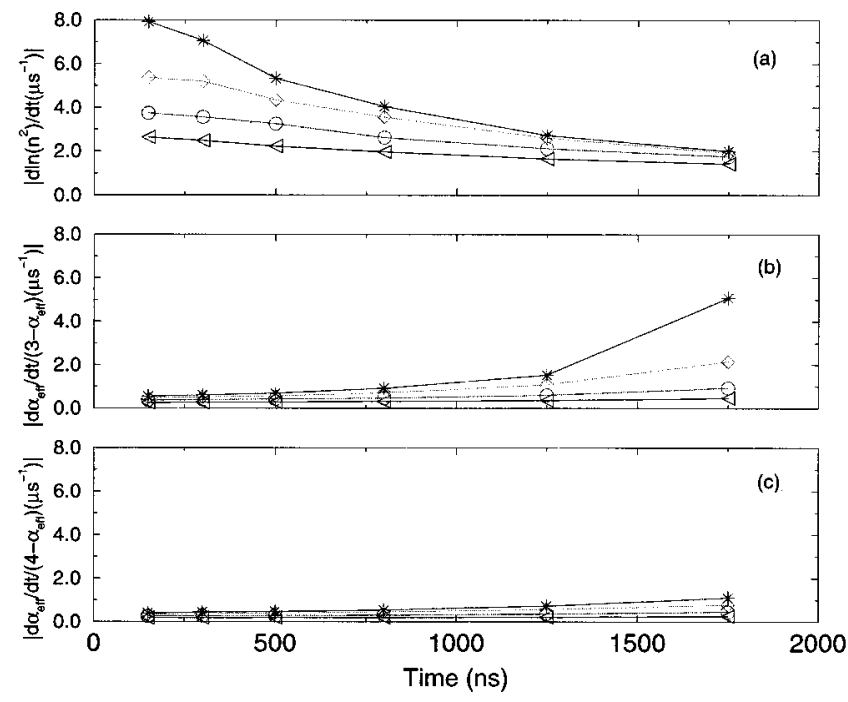

FIG. 13. (a) $\left|d \ln \left(n_{e}^{2}\right) / d t\right|$ versus time; (b) $\left|\left(d \alpha_{\text {eff }} / d t\right) /\left(k-\alpha_{\text {eff }}\right)\right|$ versus time with the assumption of $k=3 \times 10^{-6} \mathrm{~cm}^{3} / \mathrm{sec}$; (c) $\mid\left(d \alpha_{\text {eff }} / d t\right) /\left(k-\alpha_{\text {eff }} \mid\right.$ versus time with the assumption of $k=4 \times 10^{-6} \mathrm{~cm}^{3} / \mathrm{sec}$. The data are under conditions of $4 \mathrm{~mJ} / \mathrm{cm}^{2}$ laser fluence with TMAE pressures of $(*) 16$ mTorr, $(\diamond) 8$ mTorr, $(\bigcirc) 4$ mTorr, and $(\triangleleft) 2$ mTorr.

to predict from this work, but this estimate of a lower bound for the $\alpha$ value is sufficient to verify the assumption in Eq. (12).

The $\left(d \alpha_{\text {eff }} / d t\right) /\left(\alpha-\alpha_{\text {eff }}\right)$ values can be calculated under the assumption $\alpha=3 \times 10^{-6} \mathrm{~cm}^{3} / \mathrm{sec}$ or $\alpha=4 \times 10^{-6}$ $\mathrm{cm}^{3} / \mathrm{sec}$. Those results compared with the $d \ln \left(n_{e}^{2}\right) / d t$ values are shown in Fig. 13, which verifies that Eq. (12) is well satisfied for most cases, especially for higher $\alpha$ values, or at earlier times. The physical meaning for the condition implied by Eq. (12) is that $\alpha-\alpha_{\text {eff }}$ varies more slowly in time when compared to the rapidly decaying electron density.

Under the conditions of Eq. (12), the relaxation times $\tau$ of the delayed ionization can be calculated from Eq. (9), and the results are shown in Fig. 14. The points on the solid lines

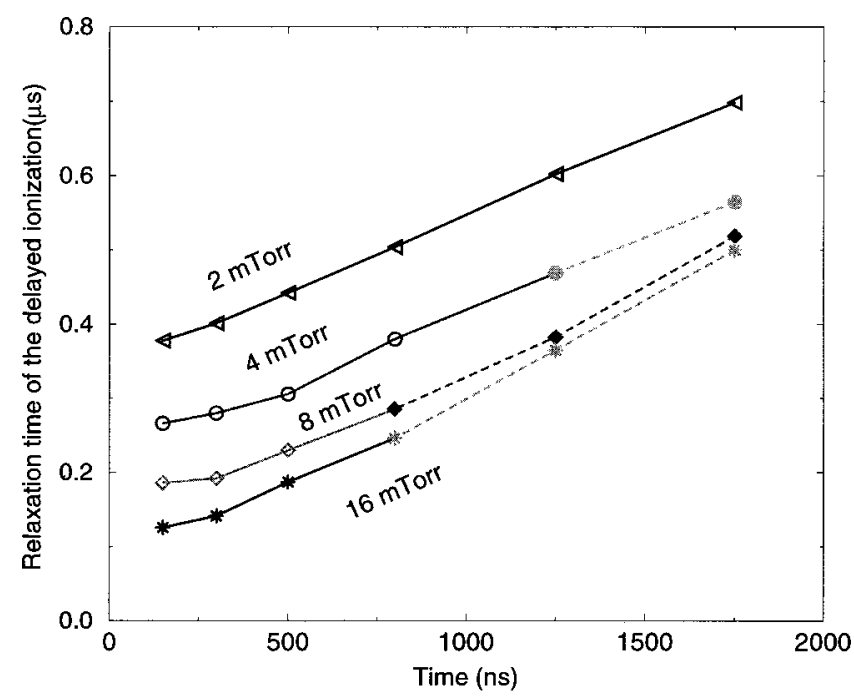

FIG. 14. The relaxation times of the delayed ionization calculated from Eq. (9). The data are under conditions of $4 \mathrm{~mJ} / \mathrm{cm}^{2}$ laser fluence with TMAE pressures of $(*) 16$ mTorr, $(\diamond) 8$ mTorr, $(\bigcirc) 4$ mTorr, and $(\triangleleft) 2$ mTorr. are under the condition that $\left(d \alpha_{\text {eff }} / d t\right) /\left(\alpha-\alpha_{\text {eff }}\right)$ is less than $30 \%$ of the $d \ln \left(n_{e}^{2}\right) / d t$ values when $\alpha=3 \times 10^{-6} \mathrm{~cm}^{3} / \mathrm{sec}$ (the lower bound) is assumed. The points on the dashed lines do not satisfy this condition, but Eq. (12) could still hold if the $\alpha$ value is larger than $3 \times 10^{-6} \mathrm{~cm}^{3} / \mathrm{sec}$, which is quite possible.

Two conclusions can be drawn from the relaxation time results from Fig. 14: (1) the relaxation time of the delayed ionization increases with time, and (2) the relaxation time of the delayed ionization decreases with increasing TMAE pressure.

The relaxation time of the delayed ionization, $\tau$, is quite comparable to the lifetime of the TMAE** state, because TMAE** $^{*}$ is the source of the delayed ionization. The lifetime of TMAE** can be influenced by the TMAE pressure and electron density due to collisions. Thus, the relaxation time $\tau$ is related to TMAE pressures and electron densities.

As the electron density decreases with time, the rate of electron quenching of TMAE** decreases, so that the lifetime of TMAE** and the relaxation time of the delayed ionization increase with time.

As the TMAE pressure increases, the rate of TMAE molecular quenching of TMAE** increases, so that the relaxation time decreases.

In sum, with the assumption that electron density change is primarily due to electron-ion recombination and delayed ionization processes, a model is developed to calculate the relaxation time of the delayed ionization, which is longer for lower TMAE pressures and lower electron densities.

\section{SUMMARY}

A fast Langmuir probe technique is developed to diagnose the decay process of a laser-produced TMAE plasma, which has a large volume (hundreds of $\mathrm{cm}^{3}$ ), high density $\left(10^{13}-10^{12} \mathrm{~cm}^{-3}\right)$, and low electron temperature $(\sim 0.1 \mathrm{eV})$.

In this experiment, the apparent electron-ion recombination coefficient is found to increase with time, and for higher TMAE pressures, the faster the increase. But the change in the recombination coefficient is only weakly dependent on electron temperature. The optical experiments show that nitrogen can enhance the delayed emission from a TMAE Rydberg state in TMAE plasma. The analysis compared with the experiments indicates that a delayed ionization after the laser excitation is an important process in the TMAE plasma decay.

With the assumption that electron density changes are primarily due to electron-ion recombination and delayed ionization processes, a model is developed to calculate the relaxation time of the delayed ionization, which is longer for lower TMAE pressures and lower electron densities.

\section{ACKNOWLEDGMENTS}

This work was primarily supported by Air Force Office of Scientific research grants (Grant Nos. F49620-94-1-0054 and F49620-97-1-0262) in cooperation with the Defense Department Research and Engineering Air Plasma Ramparts 
Multi University Research Initiative program. It was also supported in part by NSF Grant No. ECS-990548 and the University of Wisconsin.

${ }^{1}$ J. R. Woodworth, T. A. Green, and C. A. Frost, J. Appl. Phys. 57, 1648 (1985).

${ }^{2}$ G. Ding, J. E. Scharer, and K. Kelly, J. Appl. Phys. 84, 1236 (1998).

${ }^{3}$ K. Kelly, J. E. Scharer, and G. Ding, J. Appl. Phys. 85, 63 (1999).

${ }^{4}$ W. Shen, J. E. Scharer, B. G. Porter, N. T. Lam, and K. Kelly, J. Appl. Phys. 78, 6974 (1995).

${ }^{5}$ Y. S. Zhang and J. E. Scharer, J. Appl. Phys. 73, 4779 (1993).

${ }^{6}$ Y. Nakato, M. Ozaki, and H. Tsubomura, Bull. Chem. Soc. Jpn. 45, 1299 (1972).

${ }^{7}$ F. F. Chen, "Electric probes," in Plasma Diagnostic Techniques, edited by R. H. Huddlestone and S. L. Leonard (Academic, New York, 1965), Chap. 4.

${ }^{8}$ R. A. Holroyd, S. Dhrnso, and J. M. Preses, J. Phys. Chem. 89, 4144 (1985).

${ }^{9}$ A. J. T. Holmes, L. M. Lea, A. F. Newman, and M. P. S. Nightingale, Rev. Sci. Instrum. 58, 223 (1987).

${ }^{10}$ M. A. Biondi, "Recombination," in Principles of Laser Plasmas, edited by G. Bekefi (Wiley, New York, 1976), Chap. 4, pp. 127-146.

${ }^{11}$ M. T. Leu, M. A. Biondi, and R. Johnsen, Phys. Rev. A 7, 292 (1972).

${ }^{12}$ C. Huang, M. Whitaker, M. A. Biondi, and R. Johnsen, Phys. Rev. A 18, 64 (1978).

${ }^{13}$ G. Y. Marr Photoionization Processes in Gases (Academic, London, 1967).

${ }^{14}$ A. C. Jones, M. J. Dale, M. R. Banks, I. Gosney, and P. R. LangridgeSmith, Mol. Phys. 80, 583 (1993).

${ }^{15}$ E. B. Gallogly, Y. Bao, K. Han, H. Lin, and W. M. Jackson, J. Phys. Chem. 98, 3121 (1994).

${ }^{16}$ W. G. Scherzer, H. L. Selzle, E. W. Schlag, and R. D. Levine, Phys. Rev. Lett. 72, 1435 (1994).
${ }^{17}$ R. G. Neuhauser, K. Siglow, and H. J. Neusser, J. Chem. Phys. 106, 896 (1997).

${ }^{18}$ A. Held, L. Y. Baranov, H. L. Selzel, and E. W. Schlag, J. Chem. Phys. 106, 6848 (1997).

${ }^{19}$ W. G. Scherzer, H. L. Selzle, and E. W. Schlag, Z. Naturforsch. A 48, 1256 (1993).

${ }^{20}$ C. E. Alt, W. G. Sherzer, H. L. Selzle, and E. W. Schlag, Ber. Bunsenges. Phys. Chem. 99, 332 (1995).

${ }^{21}$ W. G. Sherzer, H. L. Selzle, E. W. Schlag, and R. D. Levine, Phys. Rev. Lett. 72, 1435 (1994).

${ }^{22}$ H. Krause and H. J. Neusser, J. Chem. Phys. 99, 6278 (1993).

${ }^{23}$ M. C. Cockett, H. Ozeki, K. Okuyama, and K. Kimura, J. Chem. Phys. 98, 7763 (1993)

${ }^{24}$ D. Tanaka, S. Sato, and K. Kimura, Chem. Phys. 239, 437 (1998).

${ }^{25}$ L. A. Pinnaduwage and Y. Zhu, Chem. Phys. Lett. 277, 147 (1997)

${ }^{26}$ C. C. Pan, C. C. Chou, C. H. Lu, Y. Tai, and K. C. Lin, J. Chem. Phys. 107, 3797 (1997)

${ }^{27}$ A. Amrein, R. Simpson, and P. Hackett, J. Chem. Phys. 95, 1781 (1991).

${ }^{28}$ A. Amrein, R. Simpson, and P. Hackett, J. Chem. Phys. 94, 4663 (1991).

${ }^{29}$ H. Lin, K. L. Han, Y. H. Ban, E. B. Gallogly, and W. M. Jackson, J. Phys. Chem. 98, 12495 (1994).

${ }^{30}$ K. W. Kennedy and O. Echt, J. Phys. Chem. 97, 7088 (1993).

${ }^{31}$ F. Negri and M. Z. Zgierski, J. Chem. Phys. 107, 4827 (1997).

${ }^{32}$ F. Merkt and R. N. Zare, J. Chem. Phys. 101, 3495 (1994).

${ }^{33}$ E. W. Schlag and R. D. Levine, J. Phys. Chem. 96, 10608 (1992).

${ }^{34}$ R. D. Levine, Adv. Chem. Phys. 101, 625 (1997).

${ }^{35}$ H. Nakamura, Int. Rev. Phys. Chem. 10, 123 (1991).

${ }^{36}$ E. W. Schlag, ZEKE Spectroscopy (Cambridge U.P., Cambridge, 1998).

${ }^{37}$ A. Remacle and R. D. Levine, Phys. Lett. A 173, 283 (1993).

${ }^{38}$ H. Koizumi, J. Chem. Phys. 95, 5846 (1991).

${ }^{39}$ M. Hori, K. Kimura, and H. Tsubomura, Spectrochim. Acta, Part A 24, 1397 (1967)

${ }^{40}$ Y. Nakato and H. Tsubomura, J. Lumin. 76, 2105 (1976).

${ }^{41} \mathrm{H}$. Weingarten and W. A. White, J. Org. Chem. 31, 3427 (1966). 